\title{
Steepened generalization gradients after massed extinction to the CS
}

\section{Eliot Hearst, NATIONAL INSTITUTE OF MENTAL HEALTH Hoger Poppen, STANFORD UNIVERSITY}

\begin{abstract}
Abstraet
Pigeons received intermittent reinforcement for pecking at a vertical-line stimulus. Ss were then tested for generalization to other line orientations under one of the following conditions: (a) $20 \mathrm{~min}$. of massed extinction to the vertical line, followed by a standard generalization test; (b) 20 min. of massed extinction to a horizontal line, followed by the generalization test; or (c) generalization test without any prior extinction. Both pre-extinguished groups showed steeper gradients than the group that had not been extinguished.

\section{Problem}

A popular technique for obtaining stimulus generalization gradients involves the presentation of several different stimulus values to individual Ss during extinction of the conditioned response. The results of such tests are complicated by the fact that gradients of relative generalization become reliably steeper as extinction progresses (e. g., Thomas \& Barker, 1964; Friedman \& Guttman, in press). Therefore the steepness of gradients depends to some extent upon the duration of the generalization test. Many writers interpret this progressive steepening to mean that the slope of separate extinction curves for different test stimuli varies with the proximity of the stimulus to the CS; the rate of extinction is presumably more rapid at stimuli far from CS than at values close to CS.

There is, however, another possibility to be considered in analyzing these results. Perhaps progressive steepening is due to general effects of extinction and would occur even if a variety of different stimuli were not presented during extinction. This possibility could

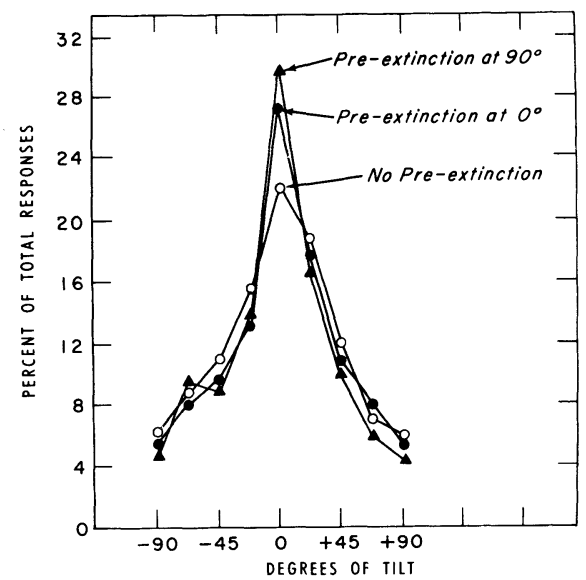

Fig. 1. Gradients of relative generalization for the three experimental groups. be tested by extinguishing responses at one stimulus value for a prolonged period before administering the standard generalization test. If such a period of massed extinction prior to generalization testing produces gradients that are reiliably steeper than those without prior extinction, it would suggest that the opportunity for Ss to compare stimuli during testing, and thus to extinguish at differential rates to the various test stimuli, is not critical in accounting for the steepeningin-extinction effect.

Therefore, in this experiment, gradients for two groups of Ss, each given massed extinction immediately before standard generalization testing, were compared with gradients from a group that received no preextinction.

\section{Method}

The Ss were 30 experimentally naive White King hen pigeons, maintained throughout the study at $75 \%$ of their free-feeding weights. Details of the key pecking apparatus, the method of varying line tilt, and the general procedure have been described in an earlier report (Hearst et al., 1964).

Fourteen days of training on a 1-min. variable interval (VI) schedule for grain reinforcement preceded generalization test days. Throughout these training periods, and all subsequent tests, $30-\mathrm{sec}$. stimulus-on periods alternated with $10-\mathrm{sec}$. stimulus-off (blackout) periods. During stimulus-on periods the CS (vertical line) was projected on the key and VI reinforcement was possible. During stimulusoff periods the chamber was dark and no reinforcement was possible. All sessions lasted 50 min.

The Ss were randomly assigned to three different experimental groups, each with $\mathrm{N}=10$, for generalization testing on Day 15. On that day each $S$ was first given a 10-min. warmup period under the same reinforcement conditions as before. This warmup period was immediately followed by one of three experimental treatments, depending on the group to which each $\mathrm{S}$ had been assigned: For Group 1 , there was a $20-\mathrm{min}$. period during which the vertical line continued to appear on the key during stimulus-on periods but no key pecks were reinforced. This period of massed extinction was immediately followed by a standard generalization test. The treatment for Group 2 was exactly the same as for Group 1 except that during massed extinction a horizontal line was projected on the key. Group 3 received a standard generalization test without any previous period of massed extinction.

Eight different orientations of the stimulus line were presented in a mixed order during generalization tests. There were 10 presentations of each line orientation for a total of 80 stimulus-on periods. Ss could not obtain any reinforcement during generalization tests.

\section{Results}

Gradients of relative generalization are shown in Fig. 1. On the abscissa the CS is designated as $0^{\circ}$ and the horizontal line as $+90^{\circ}$ and $-90^{\circ}$, as is conventional in studies employing the line-tilt dimension.

The groups that had been extinguished at either the $0^{\circ}$ or $90^{\circ}$ stimulus both showed steeper generalization gradients than the group that had received no prior extinction. Analysis of variance of the individual values that entered into the computation of these relative 


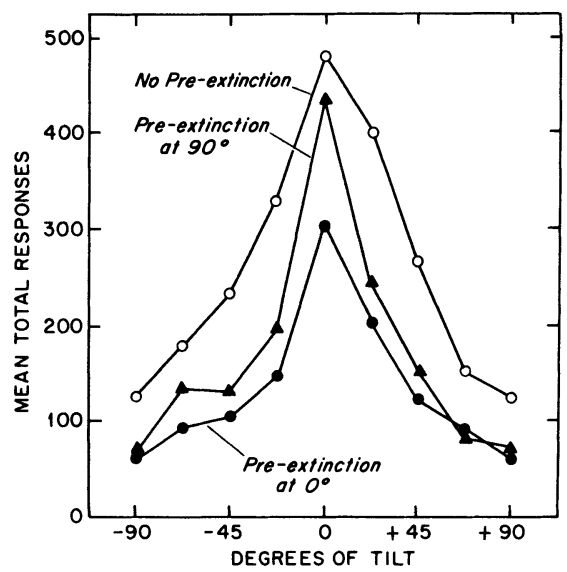

Fig. 2. Gradients of absolute generalization for the three experimental groups.

gradients revealed a significant interaction between "stimuli" and "extinction treatment" ( $F=2.56$; $\mathrm{df}=14 / 189 ; \mathrm{p}<.01$ ), which supports the conclusion that generalization gradients are reliably steeper following a period of massed extinction.

In an additional analysis, an index of gradient slope (Thomas \& Barker, 1964) was obtained for each individual $S$ in the experiment by calculating the percent of each S's total generalization test responses that occurred to the CS (i. e., Responses to the $0^{\circ}$ Stimulus/ Total Responses to All Eight Stimuli). When these indices were analyzed between groups by appropriate MannWhitney U Tests, differences between the "no preextinction" group and either of the two extinguished groups were significant at beyond the .02 level (2-tail test). Slope differences between the two extinguished groups were not significant $(U=38)$.

Figure 2 displays the absolute response frequencies from which the relative gradients of Fig. 1 were derived. The group that had been pre-extinguished at the CS emitted the fewest total responses during the test, the other extinguished group an intermediate number of responses, and the nonextinguished group the most responses. Differences in total test responses between the nonextinguished group and the group pre-extinguished at the CS were significant beyond the .02 level (MannWhitney Test), but the other group differences in total test responses did not attain an acceptable level of significance.

As would be expected, Ss given massed extinction at the CS made many more responses (Mean $=936.1$ responses) during the 20-min. period of massed extinction than Ss extinguished at the $90^{\circ}$ value (Mean $=353.1 \mathrm{re}-$ sponses). By the Mann-Whitney Test this difference was significant at beyond the .002 level.

\section{Diseussion}

This experiment showed that the gradient-steepening effect, usually observed while extinction progresses in the presence of various generalization test stimuli,. also occurs as a result of extinction to a single stimulus. Particularly interesting is the existence of this effect after massed extinction to the CS. Gradients produced by successive reinforcement and extinction at the CS are apparently steeper than those produced by reinforcement alone.

It is possible to construct hypothetical interacting gradients of excitation and inhibition around the CS which would account for the above results. For example, if one assumed that inhibitory gradients produced by extinction at the CS are much flatter than excitatory gradients produced by reinforcement at the CS, then one could predict the steepening of relative gradients obtained here.

In an experiment of Honig et al. (1959) wave length gradients following massed extinction to a non-CSvalue were compared with gradients obtained immediately after VI training; no consistent differences in slope between pre-extinguished and nonextinguished Ss were observed in that experiment. In our study, on the other hand, gradients were reliably steeper following massed extinction to the non-CS value of $90^{\circ}$. The differences in results between our experiment and that of Honig et al. may be traceable to the fact that we included a warmup period on the day of generalization testing and gave massed extinction immediately before generalization testing, whereas Honig et al. gave no pretest warmup and scheduled massed extinction on the day preceding testing. Because of the possibility of appreciable spontaneous recovery, this latter provision could have reduced the chances of obtaining an effect of pre-extinction. In addition, both (a) the inclusion of a warmup in the generalization testing procedure, and (b) the amount of delay between training and testing, have been shown to have an effect on the steepness of generalization gradients (Friedman \& Guttman, in press; Thomas \& Lopez, 1962)

\section{Referenees}

FRIEDMAN, H., \& GUTTMAN, N. A further analysis of the effects of discrimination training upon stimulus generalization. In D. Mostofsky (Ed), Stimulus generalization. Palo Alto: Stanford Univer. Press, in press.

HEARST, E., KORESKO, M., \& POPPFN, R. Stimulus generalization and the response-reinforcement contingency. J. exp. Anal. Behav., 1964, 7, 369-380.

HONIG, W. K., THOMAS, D. R., \& GUTTMAN, N. Differential effects of continuous extinction and discrimination training on the generalization gradient. J. exp. Psychol., 1959, 58, 145-152.

THOMAS, D. R., \& BARKER, E. The effects of extinction and "central tendency" on stimulus generalization in pigeons. Psychon. Sci., 1964, 1, 119-120.

THOMAS, D. R., \& LOPEZ, L. J. The effects of delayed testing on generalization slope. J. comp. physiol. Psychol., 1962, 55, 541-544.

Notes

1. We acknowledge the valuable suggestions of Dr. Joseph Openshaw, Dr. Larry Stein, and Mrs. Minne B. Koresko.

2. During 1965 the first author's address is: Department of Pharmacology, Royal College of Surgeons, Lincoln's Inn Fields, London W. C. 2, England. Reprints of this article may be obtained by writing to: E. Hearst, NIMH Clinical Neuropharmacology Research Center, Wm. A. White Building, St. Elizabeths Hospital, Washington 20, D. C. 